\title{
Biliary atresia screening in Shenzhen: implementation and achievements
}

\author{
Jiachen Zheng, ${ }^{1}$ Yongqin $\mathrm{Ye}^{2}$ Bin Wang 이, ${ }^{2}$ Lihui Zhang ${ }^{3}$
}

${ }^{1}$ Shantou University Medical College, Shantou, China

${ }^{2}$ Department of General Surgery, Shenzhen Children's Hospital, Shenzhen, Guangdong, China ${ }^{3}$ Department of Traditional Chinese Medicine, Shenzhen Children's Hospital, Shenzhen, Guangdong, China

\section{Correspondence to}

Dr Bin Wang, Department of

General Surgery, Shenzhen

Children's Hospital, Shenzhen,

Guangdong, China;

szwb1967@126.com

Published Online First

9 June 2020

\section{Linked}

- http://dx.doi.org/10.1136/ archdischild-2020-319351

Check for updates

(C) Author(s) (or their employer(s)) 2020. No commercial re-use. See rights and permissions. Published by BMJ.

To cite: Zheng J, Ye Y, Wang B, et al. Arch Dis Child 2020;105:720-723.

\section{ABSTRACT}

Objective To elaborate on the implementation and achievements of a biliary atresia (BA) screening programme in Shenzhen.

Methods In 2015, we partnered with the government in Shenzhen and attached the stool colour card (SCC) to the health handbook for mothers and children. These handbooks have been distributed through official channels to every pregnant woman in Shenzhen since 1 January 2015. A total of 118 patients diagnosed with BA at Shenzhen Children's Hospital were enrolled and divided into two cohorts based on their date of diagnosis: cohort $\mathrm{A}$ before and cohort $\mathrm{B}$ after introduction of screening. The cohorts were compared to evaluate differences in age at diagnosis, jaundice-free rate, 2-year native liver survival rate and so on.

Results After the implementation of the screening programme, the age at diagnosis decreased from $81 \pm 12$ to $56 \pm 15$ days old $(p<0.05)$, the incidence of postoperative complications decreased from $58.8 \%$ to $52.6 \%(p<0.05)$, the jaundice-free rate increased from $47.1 \%$ to $54.4 \%(p<0.05)$, and the 2 -year native liver survival rate increased from $44.4 \%$ to $52.6 \%(p<0.05)$. The percentage of patients who underwent surgery increased from $68.0 \%$ to $83.8 \%(p<0.05)$.

Conclusion In Shenzhen, our screening programme led to earlier diagnoses and better prognoses. The latter resulted in an increased willingness to undergo the Kasai procedure. Through a hospital and government collaboration, this programme exerted a considerable influence, and guardians observed benefits with only a small cost of implementation. Our results may eventually help promote the widespread use of the SCC across the whole country.

\section{INTRODUCTION}

iliary atresia (BA), an idiopathic cholangiopathy with biological characteristics that can be briefly summarised as a complete obstruction of the extrahepatic bile ducts documented by cholangiography or bile duct histology, the proliferation of intrahepatic bile ducts on liver biopsy, and marked intrahepatic fibrosis at an early age, is the leading cause of paediatric end-stage liver disease and is correlated with liver transplantation. ${ }^{1}$

Typically, patients with BA show limited symptoms in the early stages with non-specific jaundice, which directly delays diagnosis and subsequent hepatic portoenterostomy (Kasai procedure). When performed within 60 days, the Kasai procedure is more effective, with a bile flow rate of $91 \%$ and a

\section{What is already known on this topic?}

- To date, many countries and areas have adopted stool colour cards for mass cohort screening and have achieved great success.

- China has a high incidence of biliary atresia; however, stool colour cards have not been popularised on a large scale in this country.

\section{What this study adds?}

The mean age at diagnosis was 25 days lower after the screening programme was introduced.

- The jaundice-free rate was $7.3 \%$ higherand the 2-year native liver survival rate was $8.2 \%$ higher after the screening programme was implemented.

- The willingness to undergo the Kasai procedure was $15.8 \%$ higher after the screening programme was implemented.

postoperative 10 -year survival rate of $73 \% .^{2}$ Data show a decline in efficacy as the age at surgery increases. The worst-case scenario without transplantation is liver failure within a year and death within 2 years. ${ }^{3}$ In mainland China, the average age the Kasai procedure is performed is approximately 79 days old.

Pale stool is easy to observe, but guardians usually lack the relevant knowledge and awareness required to recognise an abnormal stool colour. To pursue early intervention and a favourable prognosis, a stool colour card (SCC) was created. To date, many countries and areas have adopted this method for mass cohort screening and have achieved great success. ${ }^{4-6}$ Although an SCC can reveal a pale stool, this finding does not always directly indicate BA. However, identifying this abnormality could motivate guardians to bring their children to doctors for further examination, which would be helpful in diagnosing $\mathrm{BA}$ or other potential diseases.

The incidence rate of BA in China is high; however, SCC has not been popularised on a large scale in this country. In fact, to the best of our knowledge, our BA screening programme makes Shenzhen the only area in the nation where every newborn family receives SCC. In this paper, we attempted to evaluate the feasibility and practicability of this screening method in Shenzhen. In addition, with the support of the government, we were able to attach the SCC to the health handbook for mothers and children since 2015 to ensure that 


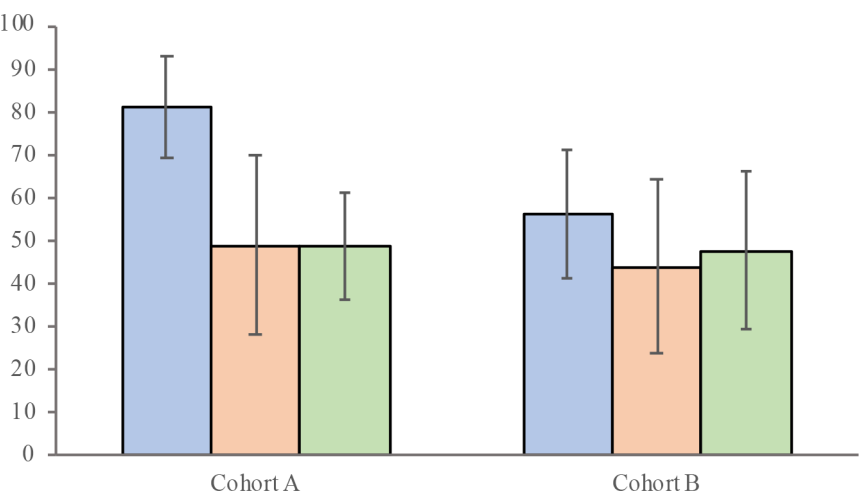

口Age at diagnosis(days) 口Length of stay(days) $\mathbf{\square}$ Medical fee(per $1000 \mathrm{RMB}$ )

Figure 1 Association between early diagnosis, length of stay and medical fee. Cohort A included patients diagnosed between 2013 and 2014 and born in Shenzhen. Cohort B included patients diagnosed between 2015 and 2017 and born in Shenzhen. RMB (¥) is China's monetary unit, and $¥ 1$ is equal to US\$0.14.

every guardian would be aware of the importance of observing stool colour. Our approach may help promote the widespread application of the SCC across the whole country.

\section{MATERIALS AND METHODS}

The health handbook for mothers and children (SCC attached) Our SCC was attached to the health handbook for mothers and children that was distributed to every mother in Shenzhen through official channels during pregnancy. These handbooks were a requisite for subsequent antenatal care and delivery as well as periodic health examinations for newborns.

The SCC included six photographs of different-coloured stool samples from normal to acholic stools. Three colours on this card were labelled as abnormal (clay-coloured, pale yellowish and light yellowish), whereas the other three colours were labelled as normal (yellowish, brown and greenish). In addition, the cards included the following: (1) instructions for guardians to continuously observe the colour of a newborn's stool after the first bowel movement; (2) information that demonstrated the association between stool colour and BA; and (3) a contact method for guardians if their infant's stool was abnormal.

\section{Patients}

A total of 118 patients diagnosed with BA by cholangiography from January 2013 to September 2017 at Shenzhen Children's Hospital were enrolled in the study. The procedures for cholangiography were as follows: under general anaesthesia, a $5 \mathrm{~mm}$ umbilical trocar was introduced via the open Hasson technique for a $30^{\circ}$ laparoscope. Carbon dioxide was insufflated to a pressure between 6 and $8 \mathrm{~mm} \mathrm{Hg}$. The liver and gall bladder were examined under direct laparoscopic vision with the operating table tilted in a $45^{\circ}$ reverse Trendelenburg position. After lifting part of the gall bladder away from the abdominal wall, a rubber tube was inserted into the gall bladder. A few millilitres of contrast agent were injected into the gall bladder. None of the patients had any other associated congenital malformations. Data on associated anomalies were not collected. All patients underwent follow-up visits through phone calls and outpatient clinic visits for 1-5 years after being discharged from the hospital.

We started to distribute our SCCs in Shenzhen on 1 January 2015; therefore, we divided the patients based on their date
Table 1 General data of the included patients

\begin{tabular}{llll}
\hline & Cohort A & Cohort B & P value \\
\hline Patients who underwent Kasai procedure & 34 & 57 & \\
Sex ratio (boys to girls) & 1.43 & 1.40 & $>0.05$ \\
Weight \pm SD $(\mathrm{kg})$ & $5.0 \pm 0.5$ & $4.7 \pm 0.8$ & $>0.05$ \\
Age at diagnosis \pm SD (days) & $81 \pm 12$ & $56 \pm 15$ & $<0.05$ \\
Age at diagnosis $\leq 60$ days & $12(35.3 \%)$ & $37(64.5 \%)$ & $<0.05$ \\
\hline
\end{tabular}

Cohort A: patients diagnosed between 2013 and 2014 and born in Shenzhen; cohort B: patients diagnosed between 2015 and 2017 and born in Shenzhen.

The measurement data are shown as $\mathrm{x} \pm \mathrm{S}$ and were compared with t-tests (two-phase) or $F$ tests (multiphase), while numerical data in each phase were described using frequency distributions and were compared with $\chi^{2}$ tests.

$\mathrm{P}<0.05$ was considered statistically significant.

of diagnosis. Shenzhen, a first-tier city in mainland China, has advanced medical resources and relatively well-educated citizens. Due to the implementation of 9-year compulsory education in China, the maternal literacy rate in Shenzhen is nearly $100 \%$. We formed two cohorts: cohort A (patients diagnosed between 2013 and 2014 and born in Shenzhen) and cohort B (patients diagnosed between 2015 and 2017 and born in Shenzhen).

\section{Evaluation index}

Age, weight, sex, age at Kasai procedure, length of stay, medical cost, jaundice clearance (total bilirubin $<20 \mathrm{mmol} / \mathrm{L}$ within 6 months of the Kasai procedure), incidence of postoperative complications, liver transplantation status and time of transplantation were recorded and compared. The cost of the surgery and hospital admission was determined as the charge made to parents for itemised care.

\section{Statistical methods}

Statistical analysis was performed using SPSS V.22.0 software. Measurement data are reported as $\mathrm{x} \pm \mathrm{s}$ and were compared with t-tests (two-phase) or $F$ tests (multiphase), while numerical data in each phase are described using frequency distributions and were compared with $\chi^{2}$ tests. $\mathrm{P}<0.05$ was considered statistically significant.

\section{RESULTS}

Age, sex and weight at Kasai procedure

The average age of the patients in cohort B who underwent surgery was $56 \pm 15$ days old, which was dramatically younger than the corresponding age of the patients in cohort $\mathrm{A}$, which was $81 \pm 12$ days old. A total of $64.5 \%$ of the patients in cohort $\mathrm{B}$ were diagnosed within 60 days, while the corresponding proportion for patients in cohort A was $35.3 \%$. The sex ratio (boys to girls) was approximately 1.4, and there was no difference between the cohorts. There was no significant difference in weight between the cohorts, although weight can be affected by BA-induced eccyliosis (table 1 and figure 1).

\section{Outcomes of the Kasai procedure}

The rate of jaundice clearance and the 2-year native liver survival rate were $54.4 \%$ and $52.6 \%$, respectively, in cohort B and $47.1 \%$ and $44.1 \%$ in cohort A. All operations were performed by the same surgical group, which consisted of surgeons from the Department of Hepatobiliary Surgery of Shenzhen Children's Hospital. Cohort B had a lower rate of postoperative complications $(52.6 \%)$ than cohort A $(58.8 \%$; table 2$)(\mathrm{p}<0.05)$. 
Table 2 Influence of the screening programme

\begin{tabular}{|c|c|c|c|}
\hline & Cohort A & Cohort B & $P$ value \\
\hline Number of biliary atresia cases & 50 & 68 & \\
\hline $\begin{array}{l}\text { Patients who underwent Kasai } \\
\text { procedure }\end{array}$ & $34(68.0 \%)$ & $57(83.8 \%)$ & $<0.05$ \\
\hline Age at diagnosis $\pm S D$ (days) & $81 \pm 12$ & $56 \pm 15$ & $<0.05$ \\
\hline Length of stay $\pm \mathrm{SD}$ (days) & $49 \pm 21$ & $44 \pm 20$ & $<0.05$ \\
\hline Medical fee \pm SD ( $¥)$ & $48850 \pm 12387$ & $47752 \pm 18121$ & $<0.05$ \\
\hline Jaundice-free & $16(47.1 \%)$ & $31(54.4 \%)$ & $<0.05$ \\
\hline Postsurgical complications & $20(58.8 \%)$ & $30(52.6 \%)$ & $<0.05$ \\
\hline Liver transplantation & $13(38.2 \%)$ & $23(40.4 \%)$ & $>0.05$ \\
\hline 2-year native liver survival rate & 15 (44.4\%) & $30(52.6 \%)$ & $<0.05$ \\
\hline Death & $7(20.6 \%)$ & $6(10.5 \%)$ & $<0.05$ \\
\hline Overall survival & $27(79.4 \%)$ & $51(89.5 \%)$ & $<0.05$ \\
\hline
\end{tabular}

Cohort A: patients diagnosed between 2013 and 2014 and born in Shenzhen; cohort B: patients diagnosed between 2015 and 2017 and born in Shenzhen.

$¥$ is China's monetary unit, and $¥ 1$ is equal to US $\$ 0.14$.

Overall survival was the sum total of patients who survived after liver

transplantation and patients who survived with a native liver.

\section{Liver transplantation}

In patients with failed Kasai procedures or recurrent jaundice, liver transplantation was the subsequent step. A total of 36 patients underwent liver transplantation during the study. When the patients were divided into two groups by their age at time of Kasai procedure, the transplantation rate in patients $\leq 60$ days was $30.1 \%$, compared with $50.0 \%$ in patients $>60$ days (table 3).

\section{DISCUSSION}

The recommended treatment for BA is sequential: the Kasai procedure is performed with the aim of restoring biliary flow to the intestine, but if this operation fails and/or life-threatening complications such as biliary cirrhosis occur, liver transplantation may eventually be needed. Early diagnosis is essential to increase the success rate of the Kasai procedure, increase the native liver survival rate and avoid liver transplantation. Screening is undoubtedly a key component to achieving this goal.

In our hospital, all the results and options were explained to the guardians before cholangiography. If the guardians wished to pursue further treatment when BA was confirmed after cholangiography, we would directly performed the Kasai procedure. If the guardians did not want further treatment, we ended the procedure right after the cholangiography. Unfortunately, quite a proportion of the guardians declined further treatment after the cholangiography even though the Kasai procedure was still practical. This phenomenon was based on complex cultural and social reasons. China is still a developing country, and due to onechild policy guardians usually have high expectations of their children; the Kasai procedure only increases the rate of native liver survival, and there is a high chance of requiring subsequent liver transplantation. The latter is not covered by the national

Table 3 Relevant factors affecting liver transplantation

$$
\text { Liver transplantation }
$$

\begin{tabular}{llll}
\cline { 2 - 4 } Kasai procedure & No $(\mathbf{n})$ & Yes $(\mathbf{n})$ & $\begin{array}{l}\text { Transplantation } \\
(\%)\end{array}$ \\
\hline Underwent Kasai procedure & 55 & 36 & 39.6 \\
Age $\leq 60$ days & 34 & 15 & 30.1 \\
Age $>60$ days & 21 & 21 & 50.0 \\
\hline
\end{tabular}

healthcare, which creates a huge burden for the family. Furthermore, preference for having a son still unfortunately exists in mainland China. Guardians also worried that even though the treatment would cost them a large sum of money, their child would still die due to late diagnosis. However, this attitude has changed a little in Shenzhen since 2015. Patients in cohort B were significantly younger at diagnosis than those in the other cohort, and this directly led to a stronger desire for surgery. As mentioned above, an earlier diagnosis is associated with less hepatic damage, which indicates a better prognosis. A total of $83.8 \%$ of the patients in cohort B underwent Kasai procedure, and this was nearly $16 \%$ higher than the rate for cohort A.

We evaluated the financial impact of this screening programme by comparing the direct medical costs of hospitalisations between the era before and the era after 2015. Early diagnosis also shortens the length of hospitalisation and reduces medical costs, which also eases the burden on guardians.

Jaundice clearance after surgery is an excellent predictor of 2-year native liver survival. A total of $54.4 \%$ of the patients in cohort $\mathrm{B}$ were jaundice-free, while the corresponding proportion of patients in cohort A was 47.1\%. Cohort B had a clearly superior 2-year native liver survival rate of $52.6 \%$, which was approximately $8 \%$ higher than that found in the other cohort. Long-term native liver survival is still being followed up. Recurrent cholangitis was the most common postsurgical complication which may require further liver transplantation. Cohort B also showed a lower rate of cholangitis. However, the difference was not remarkable. The surgeons who performed the procedures in this study had extensive experience in the Kasai procedure, and this might explain the similar incidence of postoperative complications between the cohorts.

Despite undergoing the Kasai procedure, some of the patients still needed additional intervention through liver transplantation. However, in mainland China, it is hard to obtain a matched liver, and subsequent treatment is expensive, which led to only a slight increase in the liver transplantation rate.

In 1994, Matsui and Ishikawa reported a study ${ }^{7}$ in which the SCC was used to increase the efficacy of identifying BA at the 1-month health check-up in Japan. Inspired by those results, Taiwan started a pilot screening programme in 2002 and generalised the programme nationwide in $2004 .^{8}$ Specifically, guardians were asked to report the colour of their infant's stool at 30 days of age to the paediatrician or as soon as the stool turned pale. The screening programme in Taiwan had a sensitivity of $89.7 \%$, a specificity of $99.9 \%$, a positive predictive value of $28.6 \%$ and a negative predictive value of $99.9 \% .{ }^{9}$ A subsequent study showed that there were associations between a young age at the time of surgery and both a significant reduction in hospitalisations postsurgery and a decreased mortality rate. ${ }^{10}$ The instructions were similar in Japan: mothers were asked to bring completed SCCs to their infants' paediatricians at their 1-month newborn visits. ${ }^{11}$ However, in a cohort study in Canada, the return rate of the SCCs was only $63.3 \%$, indicating that nearly half of the individuals were likely to not respond..$^{12}$ Among the non-responding individuals, half had seen and used the SCC, which covered over $80 \%$ of the population. Switzerland and the USA have also adopted similar screening strategies. ${ }^{13} 14$

Since mainland China, unlike Taiwan, Japan or Canada, does not have a reporting system, guardians are not aware that they should describe their infant's stool colour to their paediatrician. Although China is widely believed to be a high-prevalence area, $\mathrm{BA}$ is still a relatively rare condition for most infants; thus, there are limited funds and human resources available for screening, and primary care physicians have a limited ability to recognise 
BA. Additionally, mainland China has not established a functional frame of fundamental medical care. Guardians usually take their infants to different paediatricians, and this phenomenon increases the difficulty of diagnosis. In addition, assessing stool colour is also challenging for guardians and primary physicians. An early Kasai procedure is essential to the prognosis of patients with BA; however, the reality is that too much time and money are wasted in primary institutions.

In our study, we did not establish a similar reporting system. Although we did not question guardians about whether they found any abnormality based on the SCC, we noticed that the percentage of patients who were hospitalised due to palecoloured stool was higher than the percentage hospitalised for persistent jaundice. This was strong evidence indicating that our screening programme worked. Before our screening programme, persistent jaundice was the main symptom for guardians to recognise an abnormality.

The innovation in our practice is that we partnered with the government in 2015 to attach the SCC to the health handbook for mothers and children. This collaboration avoided the limitations of traditional distribution. Within mainland China, several areas, such as Zunyi or Tianjin, have also started regional screening programmes with SCCs. However, during their execution processes, the SCCs were directly distributed to the guardians by doctors or volunteers at random, causing some cohorts to be overlooked. In addition, a single small card is easy to lose, and when a card was lost few guardians asked for another one. Without the instructions on the cards, guardians tended to stop inspecting the infant's stool. In our screening programme, we were able to cover all the infants in Shenzhen without using extra human resources; additionally, we added only one page to the handbook, and this required minimal funding. Finally, the SCCs that were placed in the health handbook for mothers and children offered a reference for doctors who were not familiar with BA and raised awareness about BA in parents, since parents believe that only important information would be added to an officially issued handbook.

In conclusion, the implementation of a screening programme with SCCs in Shenzhen led to earlier diagnoses and a stronger willingness to undergo treatment and therefore better prognoses. Due to partnership with the government, the influence of this programme was substantial. The programme raised awareness among guardians and was of low cost to implement; thus, it is worthwhile to generalise this screening programme across the country.
Contributors Study conception and design: JZ, BW. Data acquisition: JZ, BW, LZ. Analysis and data interpretation: JZ, YY. Drafting of the manuscript: JZ. Critical revision: JZ, YY, LZ. Submission of the manuscript: BW.

Funding Sanming Project of Medicine in Shenzhen (grant number SZSM201812055).

Competing interests None declared.

Patient consent for publication Not required.

Ethics approval The study was reviewed and approved by the Ethics Committee of Shenzhen Children's Hospital.

Provenance and peer review Not commissioned; externally peer reviewed.

ORCID iD

Bin Wang http://orcid.org/0000-0002-0951-4652

\section{REFERENCES}

1 Lakshminarayanan B, Davenport M. Biliary atresia: a comprehensive review. J Autoimmun 2016;73:1-9.

2 Chang M-H. Screening for biliary atresia. Chang Gung Med J 2006;29:231-3.

3 Lien $\mathrm{T}-\mathrm{H}$, Chang M-H, Wu J-F, et al. Effects of the infant stool color card screening program on 5-year outcome of biliary atresia in Taiwan. Hepatology 2011;53:202-8.

4 Wang KS, Section on Surgery, Committee on Fetus and Newborn, et al. Newborn screening for biliary atresia. Pediatrics 2015;136:e1663-9.

5 Woolfson JP, Schreiber RA, Butler AE, et al. Province-wide biliary atresia home screening program in British Columbia: evaluation of first 2 years. J Pediatr Gastroenterol Nutr 2018:66:845-9.

6 Witt M, Lindeboom J, Wijnja C, et al. Early detection of neonatal cholestasis: inadequate assessment of stool color by parents and primary healthcare doctors. Eur J Pediatr Surg 2016;26:67-73.

7 Matsui A, Ishikawa T. Identification of infants with biliary atresia in Japan. Lancet 1994;343:925.

8 Lee $\mathrm{M}$, Chen SC-C, Yang H-Y, et al. Infant stool color card screening helps reduce the hospitalization rate and mortality of biliary atresia: a 14-year nationwide cohort study in Taiwan. Medicine 2016:95:e3166.

9 Chen S-M, Chang M-H, Du J-C, et al. Screening for biliary atresia by infant stool color card in Taiwan. Pediatrics 2006;117:1147-54.

10 Lin J-S, Chen SC-C, Lu C-L, et al. Reduction of the ages at diagnosis and operation of biliary atresia in Taiwan: a 15-year population-based cohort study. World J Gastroenterol 2015;21:13080-6.

11 Gu Y-H, Yokoyama K, Mizuta K, et al. Stool color card screening for early detection of biliary atresia and long-term native liver survival: a 19-year cohort study in Japan. J Pediatr 2015;166:897-902.

12 Schreiber RA, Masucci L, Kaczorowski J, et al. Home-Based screening for biliary atresia using infant stool colour cards: a large-scale prospective cohort study and costeffectiveness analysis. J Med Screen 2014;21:126-32.

13 Wildhaber BE, Majno P, Mayr J, et al. Biliary atresia: Swiss national study, 1994-2004. J Pediatr Gastroenterol Nutr 2008;46:299-307.

14 Goodhue C, Fenlon M, Wang KS. Newborn screening for biliary atresia in the United States. Pediatr Surg Int 2017;33:1315-8. 